Verfahrenstechnik in Beispielen 
Josef Draxler • Matthäus Siebenhofer

\section{Verfahrenstechnik in Beispielen}

Problemstellungen, Lösungsansätze, Rechenwege

Springer Vieweg 
Josef Draxler

Montanuniversität Leoben

Weiz, Österreich
Matthäus Siebenhofer

Technische Universität Graz

Graz, Österreich

Die Deutsche Nationalbibliothek verzeichnet diese Publikation in der Deutschen Nationalbibliografie; detaillierte bibliografische Daten sind im Internet über http://dnb.d-nb.de abrufbar.

\section{Springer Vieweg}

(C) Springer Fachmedien Wiesbaden 2014

Das Werk einschließlich aller seiner Teile ist urheberrechtlich geschützt. Jede Verwertung, die nicht ausdrücklich vom Urheberrechtsgesetz zugelassen ist, bedarf der vorherigen Zustimmung des Verlags. Das gilt insbesondere für Vervielfältigungen, Bearbeitungen, Übersetzungen, Mikroverfilmungen und die Einspeicherung und Verarbeitung in elektronischen Systemen.

Die Wiedergabe von Gebrauchsnamen, Handelsnamen, Warenbezeichnungen usw. in diesem Werk berechtigt auch ohne besondere Kennzeichnung nicht zu der Annahme, dass solche Namen im Sinne der Warenzeichen- und Markenschutz-Gesetzgebung als frei zu betrachten wären und daher von jedermann benutzt werden dürften.

Lektorat: Dr. Daniel Fröhlich, Pamela Frank

Gedruckt auf säurefreiem und chlorfrei gebleichtem Papier

Springer Vieweg ist eine Marke von Springer DE. Springer DE ist Teil der Fachverlagsgruppe Springer Science+Business Media.

www.springer-vieweg.de 


\section{Vorwort}

Dieses Buch ist nicht als Lehrbuch, sondern als Ergänzung zu Lehrbüchern der Verfahrenstechnik konzipiert. In jedem Kapitel sind zunächst die wichtigsten Gesetzmäßigkeiten des entsprechenden Themenbereiches zusammengefasst, welche anschließend durch zahlreiche praxisnahe Beispiele verdeutlicht werden.

In unserer mehr als dreißigjährigen Lehrtätigkeit haben wir immer wieder die Bedeutung des eigenständigen Bearbeitens und Lösens von Beispielen feststellen können. Es ist selten ausreichend, die Beispiele nur durchzulesen und sich einen ungefähren Lösungsweg zu überlegen. Erst durch das konkrete, selbständige Rechnen kann überprüft werden, ob das Themengebiet beherrscht wird und für andere Aufgabenstellungen angewandt werden kann.

Für die Lösung der Beispiele wird folgende Vorgangsweise empfohlen:

1. Zeichnen einer Prinzipskizze mit Benennung aller bekannten und gesuchten Größen; ist bei einigen Beispielen gegeben, bei den meisten aber selbst zu erstellen.

2. Anschreiben aller Annahmen und Vereinfachungen.

3. Aufstellen der benötigen Gleichungen.

4. Lösen der Gleichungen.

5. Diskussion der Ergebnisse.

Für die Berechnung vieler Beispiele ist die Verwendung eines Computers mit entsprechender Mathematik-Software erforderlich. Aber auch für einfache Beispiele wird die Berechnung mittels Computer empfohlen. So können damit auf einfache Weise Parameterstudien, Sensitivitätsanalysen und Animationen durchgeführt werden. Auch sind damit exakte Lösungen vielfach nicht aufwändiger als Näherungslösungen, deren Güte somit einfach überprüft werden kann.

Alle Beispiele wurden mit dem Computer-Algebra-System (CAS) Mathematica berechnet. Es muss aber betont werden, dass nicht die Verwendung dieses Programmes im Vordergrund steht, sondern das Verstehen und selbständige Lösen der Beispiele mit beliebigem Computerprogramm. Alle Mathemati$c a$-Dateien stehen auch als pdf-Dateien zur Verfügung, so dass einzelne Programmschritte und Zwischenergebnisse von allen Anwendern anderer Programme eingesehen werden können.

Etwa die Hälfte der Beispiele wurden der Literatur entnommen, im Text ist die entsprechende Quelle zitiert; genauere Angaben wie Seitenzahl, Beispielnummer oder ob das Beispiel direkt übernommen, verändert oder erweitert wurde, sind in der Mathematica-Datei bzw. in der entsprechenden pdf-Datei angegeben. Für einige Beispiele konnte die Quelle nicht mehr gefunden werden.

In ca. zehn Beispielen werden Dampftafeln gebraucht. Diese können z. B. in [1], [2], [3], [4]oder [5] gefunden werden.

Zum Gebrauch der Mathematica-Dateien:

Diese können von den Internetseiten des Springer-Verlages zu diesem Buch (springer.com) kopiert werden. Die Stoffdatendateien (.m-files) müssen in das Standardverzeichnis des MathematicaProgrammes kopiert werden (Pfad in: Directory[]), damit in den Programmen direkt darauf zugegriffen werden kann. 


\section{INHALTSVERZEICHNIS}

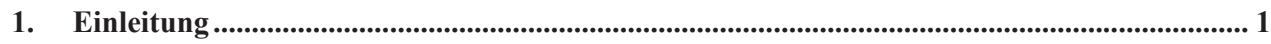

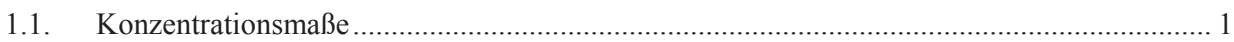

1.2. Signifikante Stellen ................................................................................................... 2

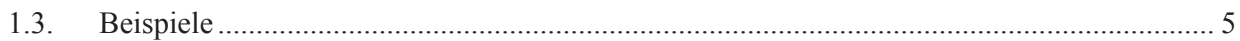

2. Thermodynamik I: Grundbegriffe .......................................................................................... 7

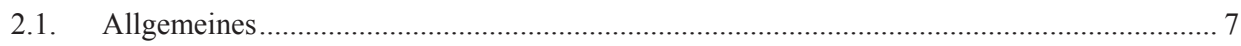

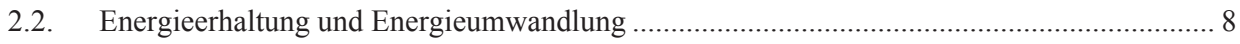

2.2.1. Erster Hauptsatz für geschlossene Systeme.................................................................... 8

2.2.2. Erster Hauptsatz für offene Systeme …................................................................. 9

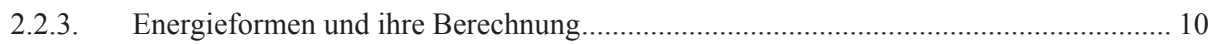

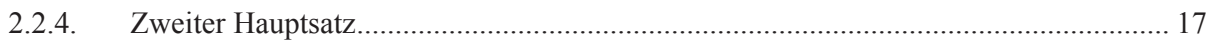

2.2.5. Zusammenstellung der Berechnungsmöglichkeiten für die Enthalpie und Entropie .... 18

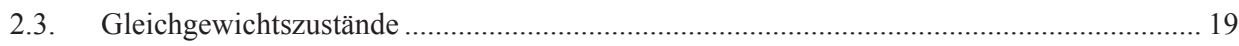

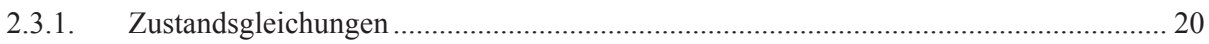

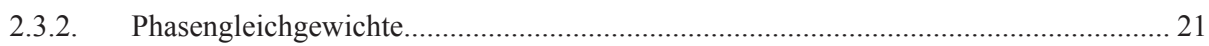

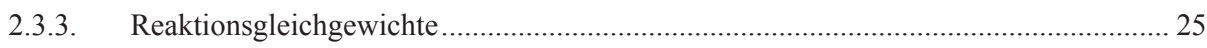

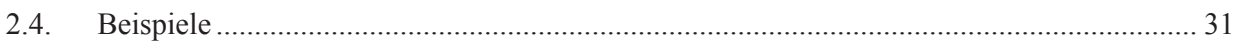

3. Grundlagen Wärme-, Stoff- und Impulstransport............................................................. 53

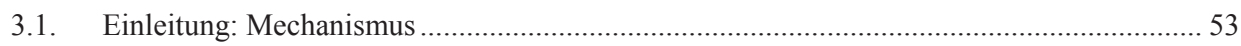

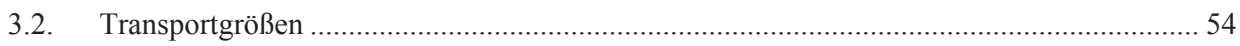

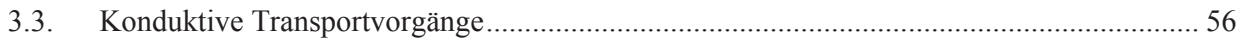

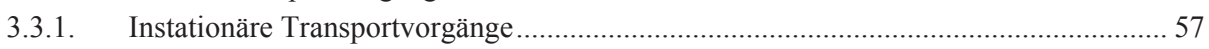

3.3.2. Transportkoeffizienten (Leitwerte) und Ausgleichskoeffizienten.................................. 57

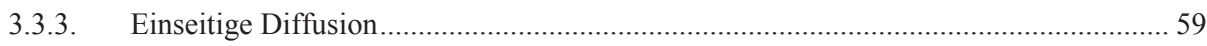

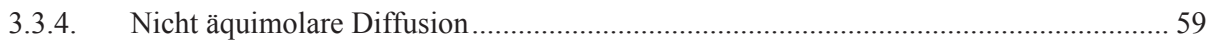

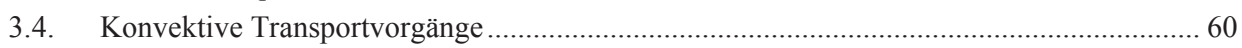

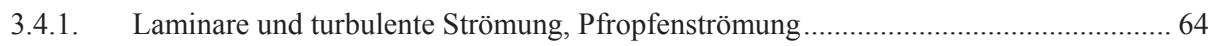

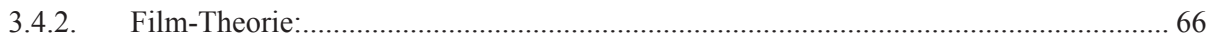

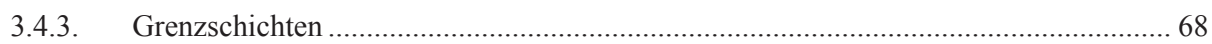

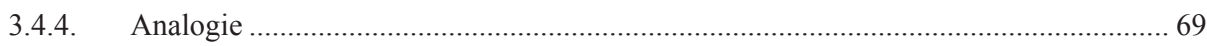

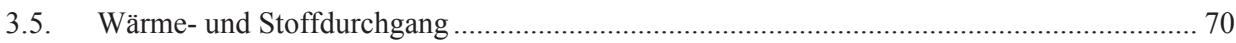

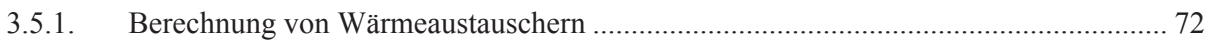

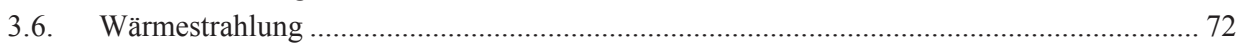

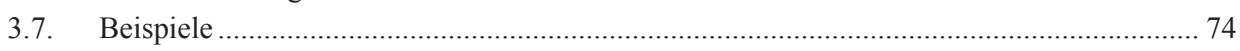

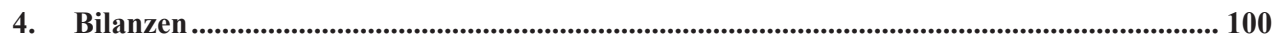

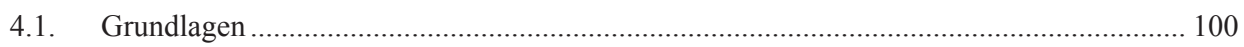

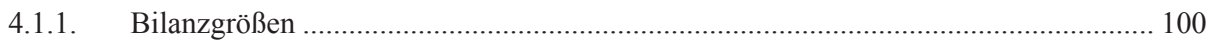

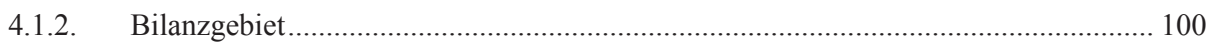

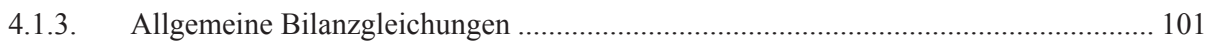

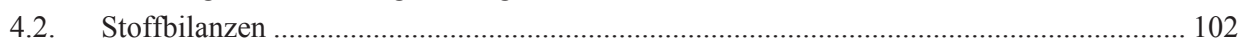

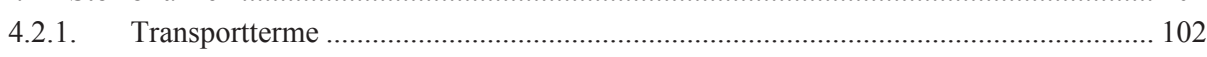

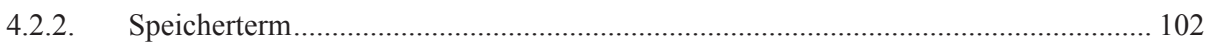

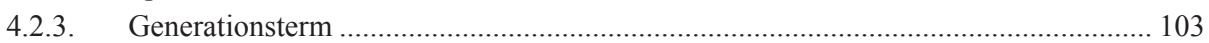

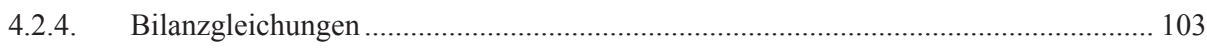




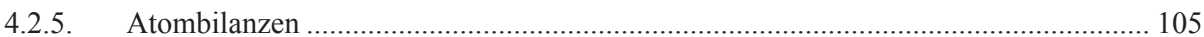

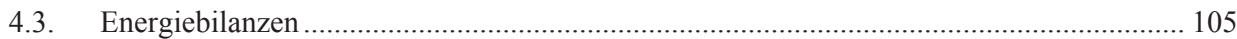

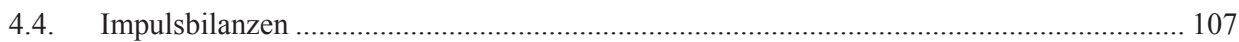

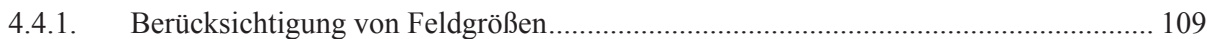

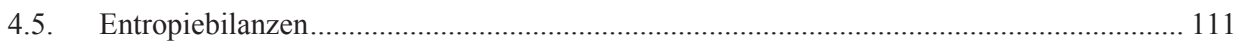

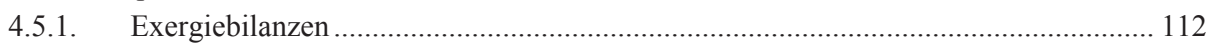

4.6. Zusammenstellung der integralen Bilanzgleichungen.................................................. 113

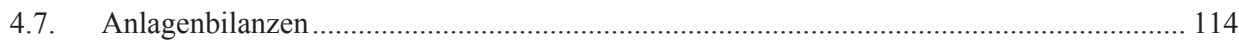

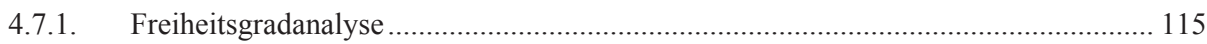

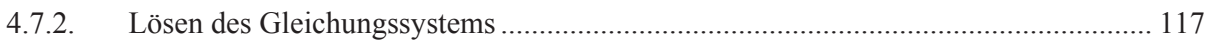

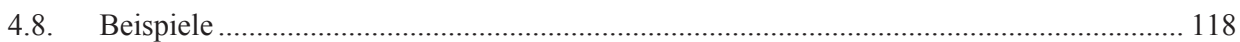

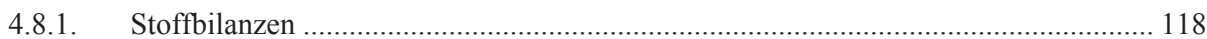

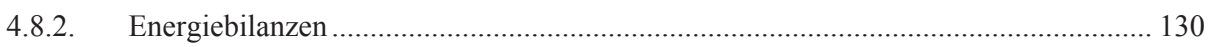

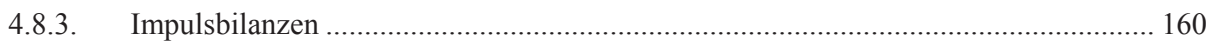

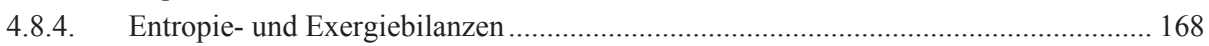

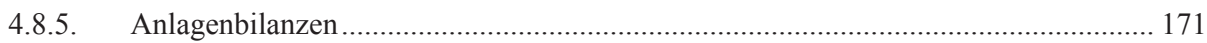

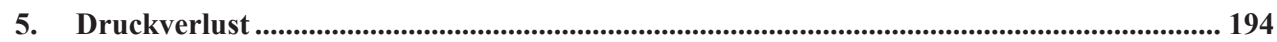

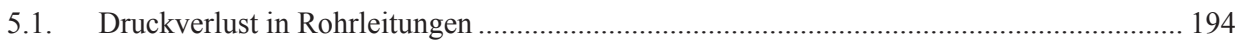

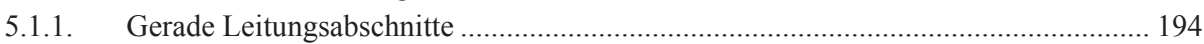

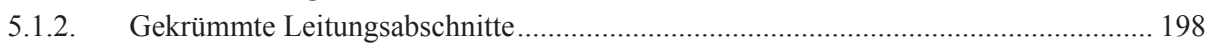

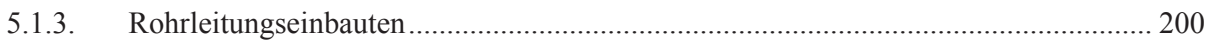

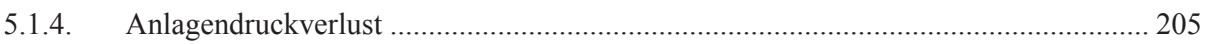

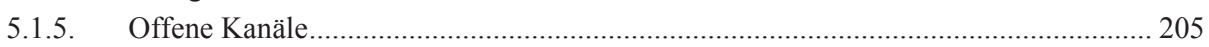

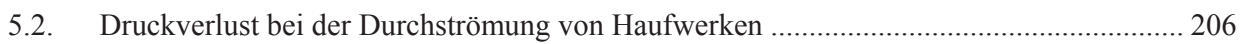

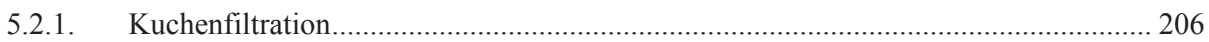

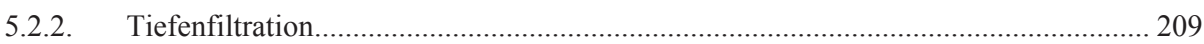

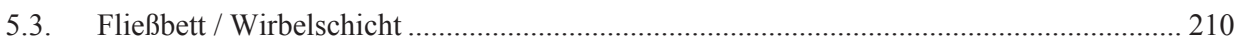

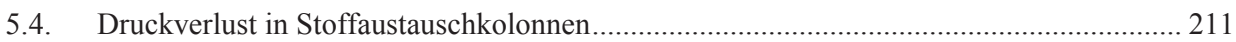

5.4.1. Druckverlust in Benetzungskolonnen........................................................................... 211

5.4.2. Druckverlust an Kolonnenböden ........................................................................... 215

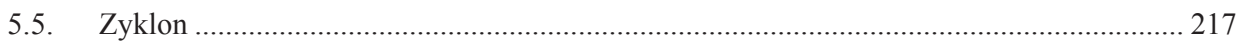

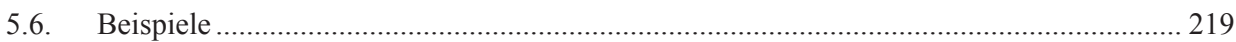

6. Partikelgrößen und Partikelgrößenverteilungen................................................................ 239

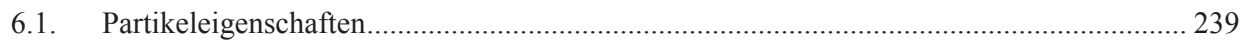

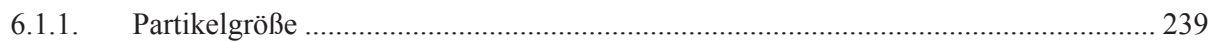

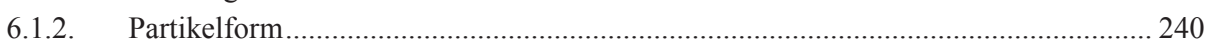

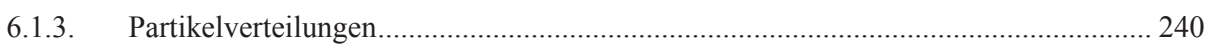

6.2. Trennen von Partikeln ................................................................................................... 245

6.2.1. Begriffe und Definitionen....................................................................................... 245

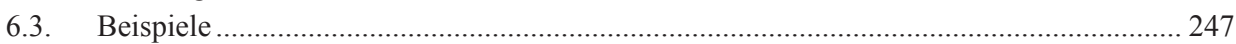

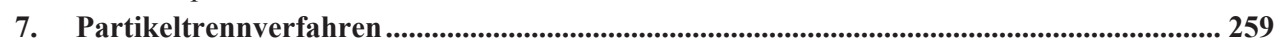

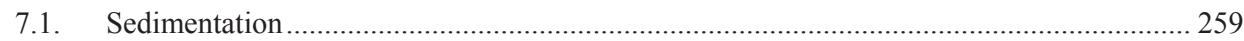

7.1.1. Stationäre Sedimentation kugelförmiger Einzelpartikel.............................................. 259

7.1.2. Stationäre Sinkgeschwindigkeit von nicht kugelförmigen Partikeln und

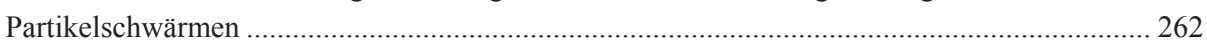

7.1.3. Auslegung Sedimentationsbecken............................................................................ 262

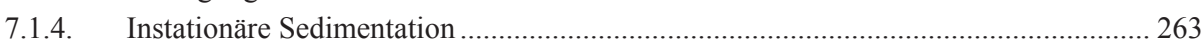




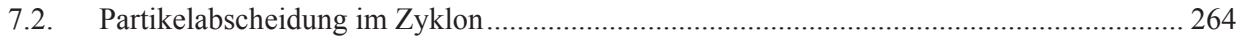

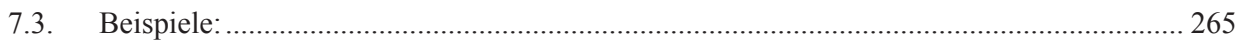

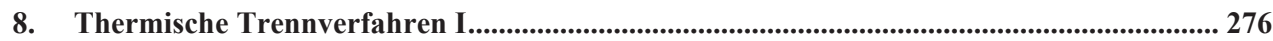

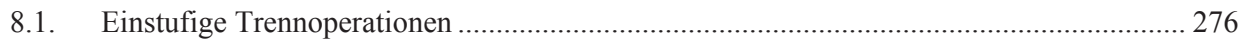

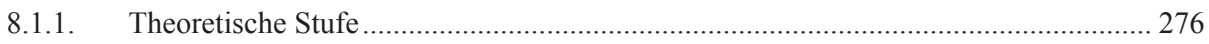

8.1.2. Gleichgewichtsgesetze für physikalisch wirkende Trennverfahren ............................. 276

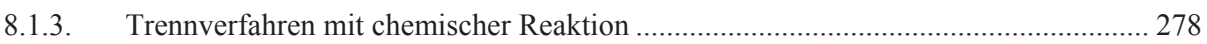

8.1.4. Beispiele zu einstufigen Trennoperationen .............................................................. 278

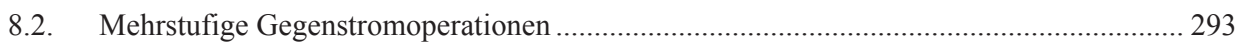

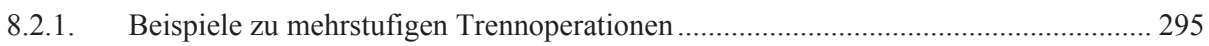

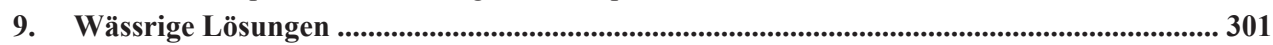

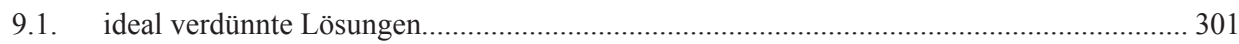

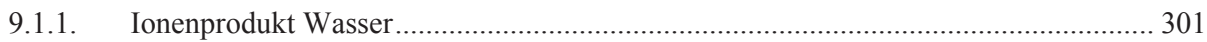

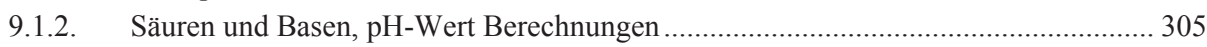

9.1.3. Lösungs- und Fällungsreaktionen, Komplexbildung...................................................... 306

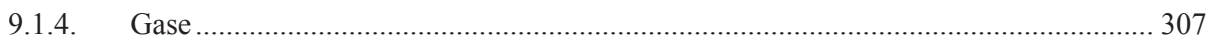

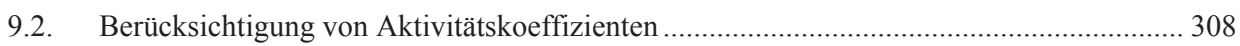

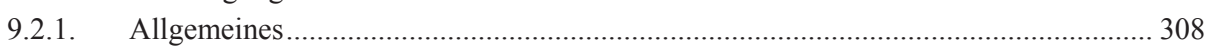

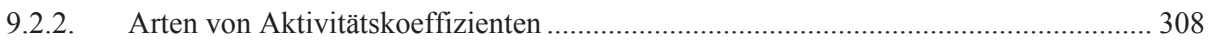

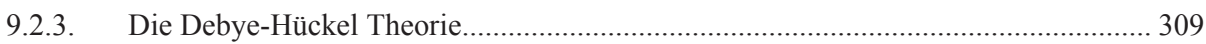

9.2.4. Erweiterung der Debye-Hückel-Theorie ohne Berücksichtigung von Nahwirkungen 310

9.2.5. Erweiterung der Debye-Hückel-Theorie mit Berücksichtigung von Nahwirkungen .. 311

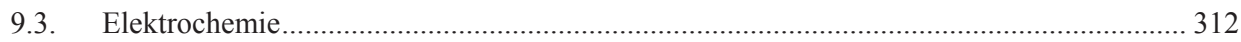

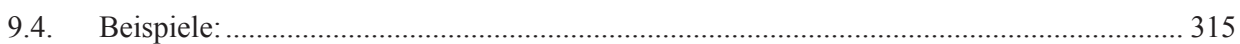

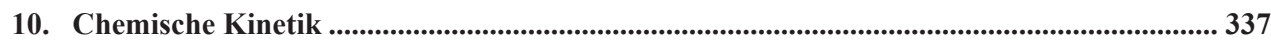

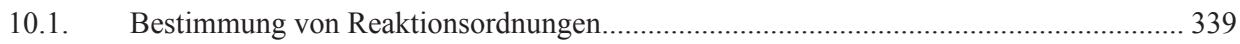

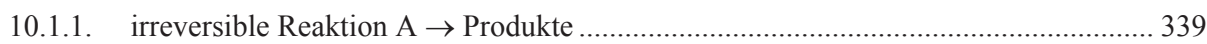

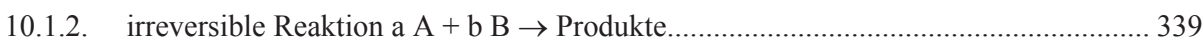

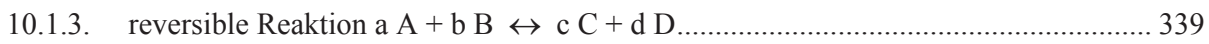

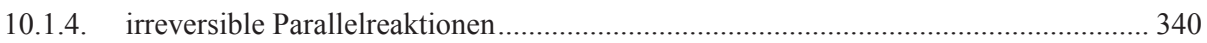

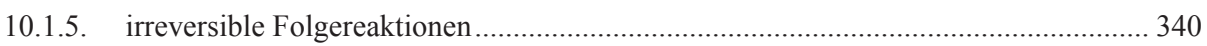

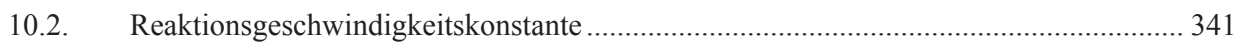

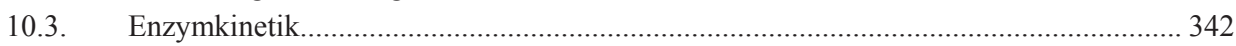

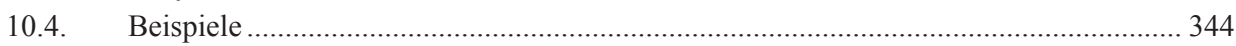

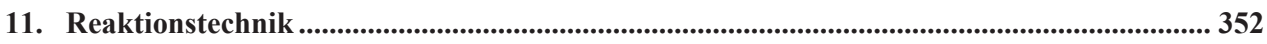

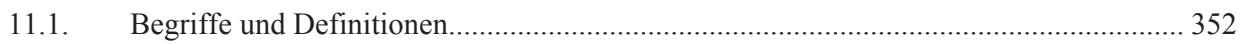

11.2. Bilanzgleichungen für isotherme ideale Reaktoren..................................................... 353

11.2.1. Diskontinuierlicher Rührkessel ................................................................................. 353

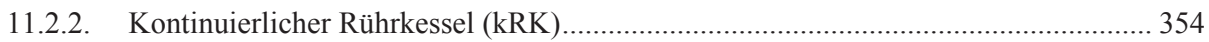

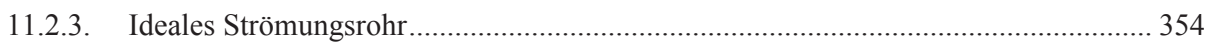

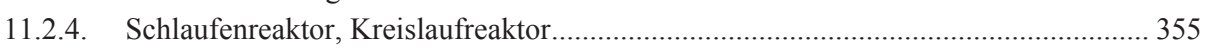

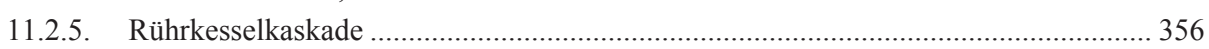

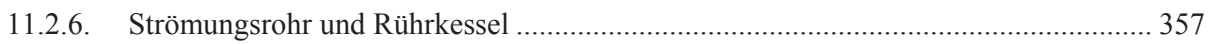

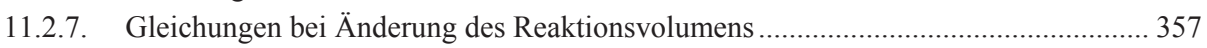

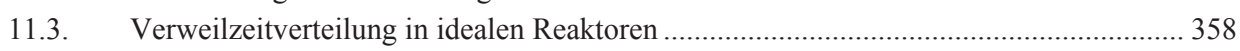

11.3.1. Kenngrößen der Verweilzeitverteilungen...................................................................... 358 


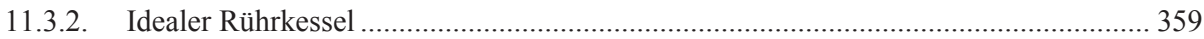

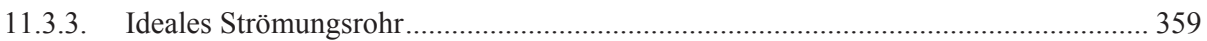

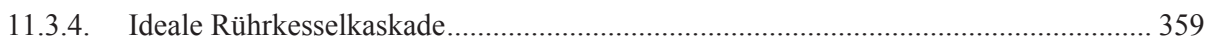

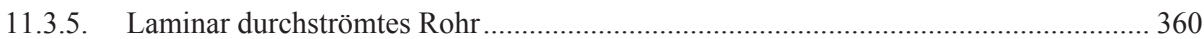

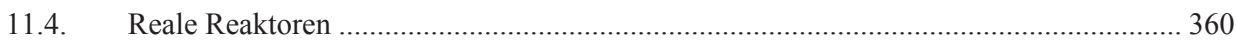

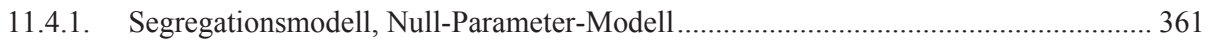

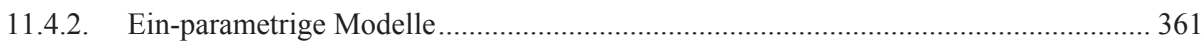

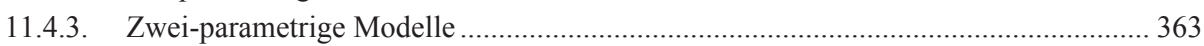

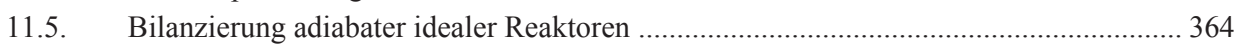

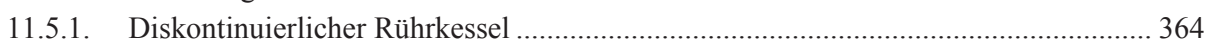

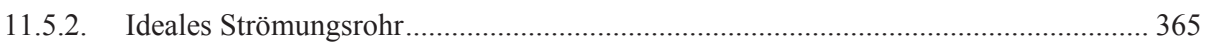

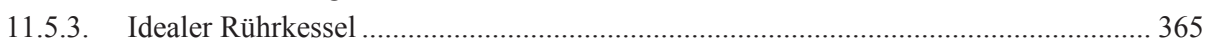

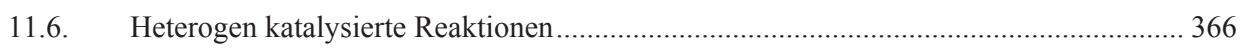

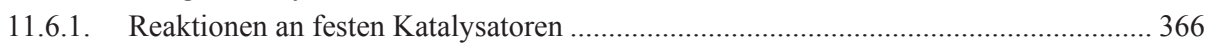

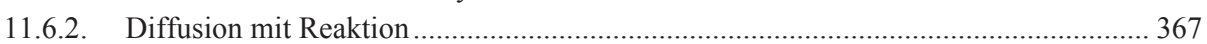

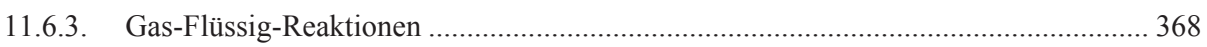

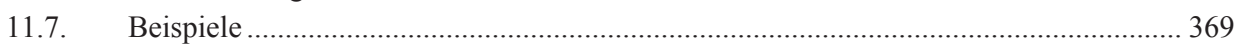

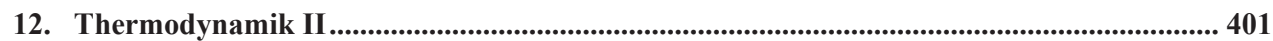

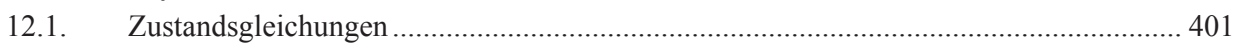

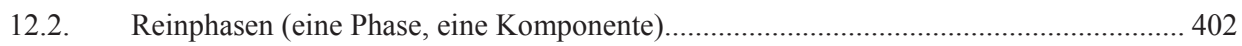

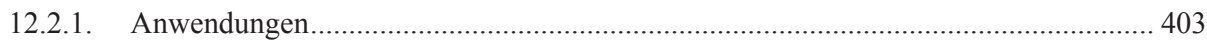

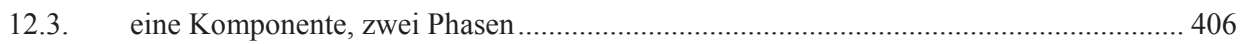

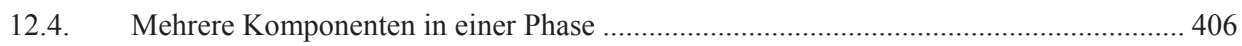

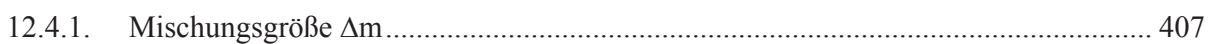

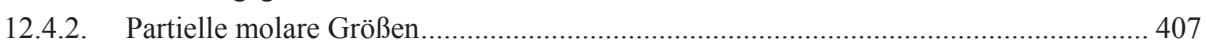

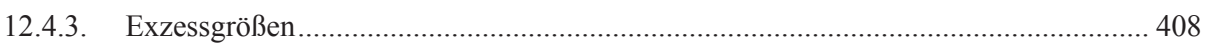

12.5. Mehrere Komponenten in zwei Phasen ............................................................................ 410

12.5.1. Berechnung des unsymmetrischen Aktivitätskoeffizienten.......................................... 411

12.5.2. Berechnung des symmetrischen Aktivitätskoeffizienten .............................................. 411

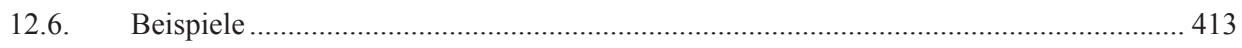

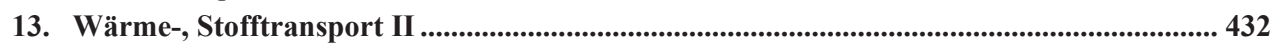

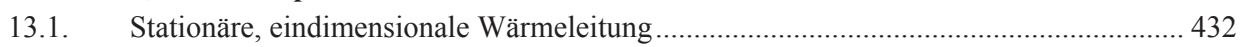

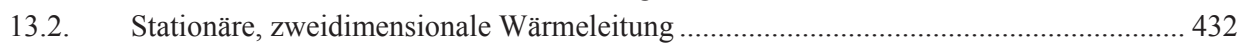

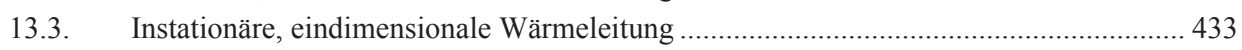

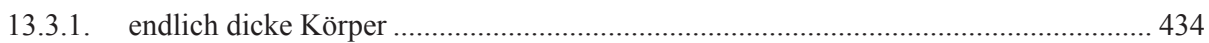

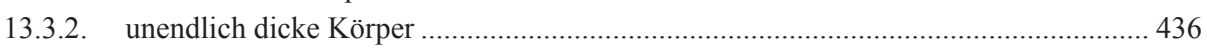

13.4. Numerische Lösungen der Wärmetransportgleichungen ............................................... 437

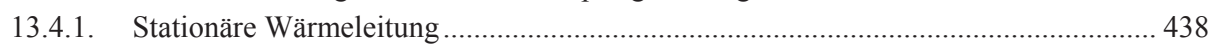

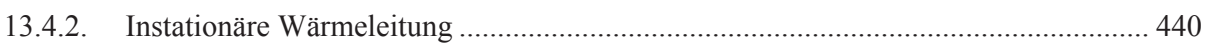

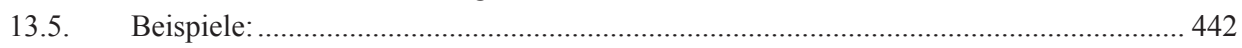

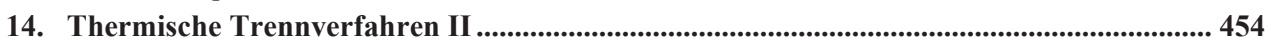

14.1. Berechnung der Kolonnenhöhe mit dem Stoffaustauschkonzept........................................ 454

14.1.1. Stoffaustausch mit chemischer Reaktion.......................................................................... 455

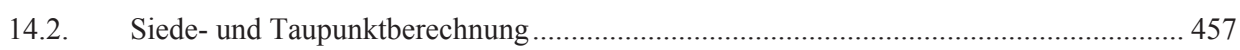

14.2.1. Eine Komponente in Flüssig- und Dampfphase ............................................................. 457

14.2.2. Eine Komponente in Flüssigphase, zwei Komponenten in Gasphase .......................... 458

14.2.3. Zwei Komponenten in Flüssigphase, eine in Dampfphase......................................... 459 
14.2.4. Zwei oder mehr Komponenten in Flüssig- und Gasphase............................................ 459

14.2.5. Zwei Komponenten, Gasphase und zwei flüssige Phasen........................................... 459

14.2.6. Drei Komponenten, eine Gasphase und zwei flüssige Phasen .................................... 460

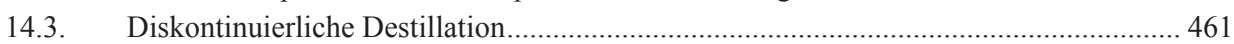

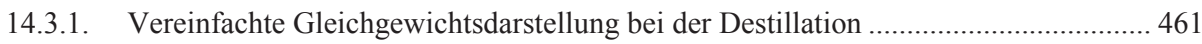

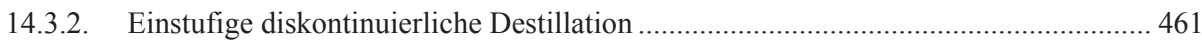

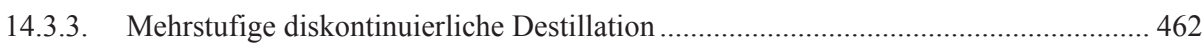

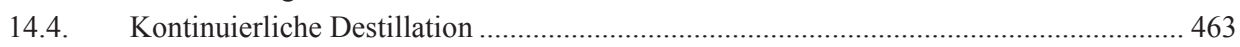

14.4.1. Berechnung der Stufenzahl ohne Wärmebilanz ............................................................. 464

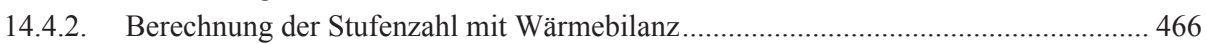

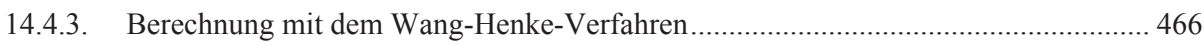

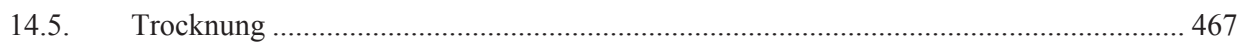

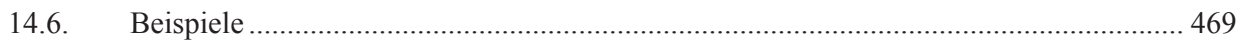

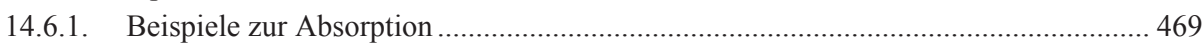

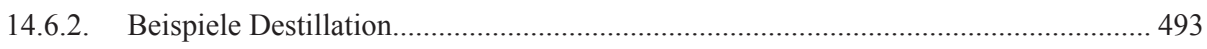

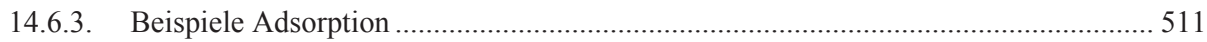

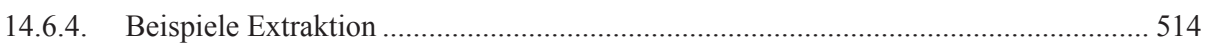

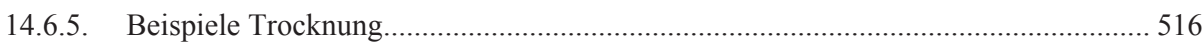

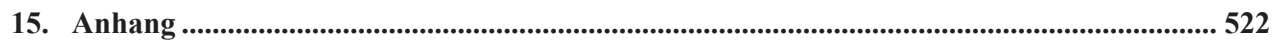

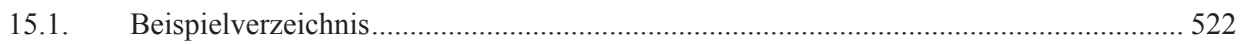

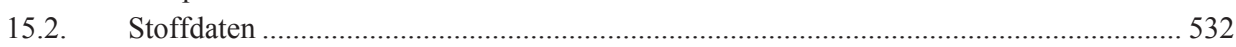

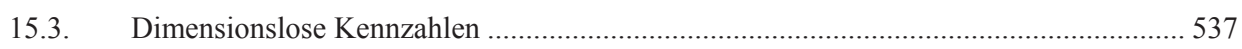

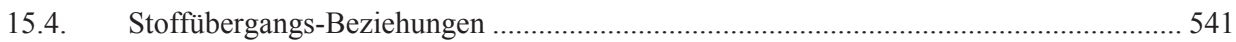

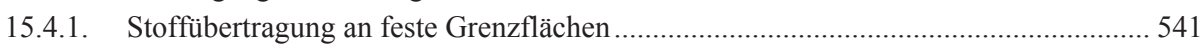

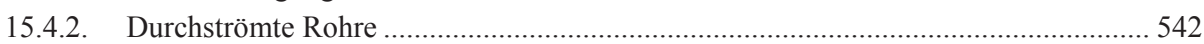

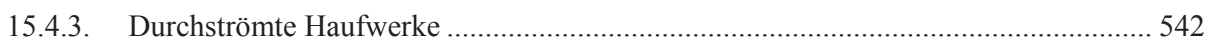

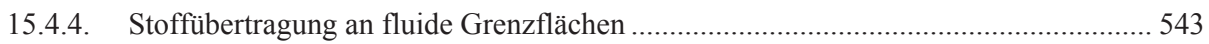

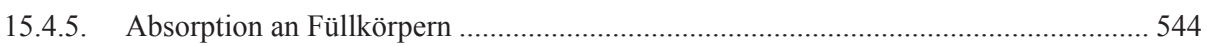

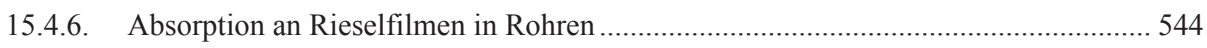

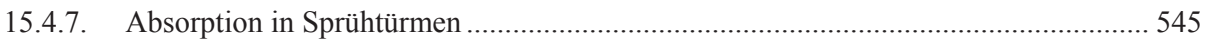

15.4.8. Auflösen und Kristallisieren in Rührkesseln .............................................................. 545

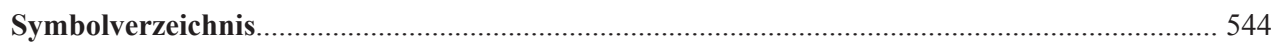

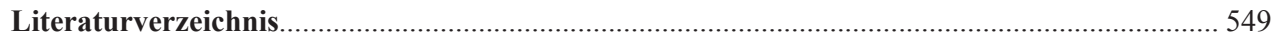

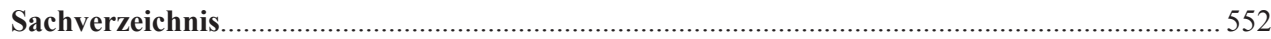

